\title{
Comparação das comunidades de Entomobryidae e Isotomidae (Collembola) entre plantio direto em três níveis de fertilidade, plantio convencional e um ecossistema natural (campo nativo) em Ponta Grossa, Paraná, Brasil
}

\author{
Klaus Dieter Sautter ${ }^{1}$ \\ Honório Roberto dos Santos ${ }^{2}$ \\ Paulo Justiniano Ribeiro Júnior ${ }^{3}$
}

\begin{abstract}
Comparison of the communities of Entomobryidae and Isotomidae (Collembola) among no-tillage in three levels of fertility, conventional tillage and a natural ecosystem (native grassland) in Ponta Grossa, Paraná, Brazil. This work had as objective to compare the communities of Entomobryidae and Isotomidae (Collembola) among no-tillage in three fertility levels, conventional tillage and a natural ecosystem (native grassland). In the no-tillage in low fertility and medium fertility Entomobryidae and Isotomidae had populational picks in the winter and in the summer. In the no-tillage in area of high fertility, there was a populational pick for both families in the summer. In relation to the conventional tillage, Entomobryidae had a populational pick in the winter, and Isotomidae in the winter and in the summer. In the natural ecosystem Entomobryidae presented populational picks in the winter and in the summer and Isotomidae had regular populational fluctuation along the period of the experiment. The final mean density of Entomobryidae was larger in the natural ecosystem, proceeded by the treatments of no-tillage and finally, for the conventional tillage. In relation to Isotomidae, the no-tillage in area of low fertility was superior, coming the conventional tillage soon after, the no-tillage in medium and high fertility, and, finally, the natural ecosystem.

KEY WORDS. Collembola, Entomobryidae, Isotomidae, no-tillage, conventional tillage
\end{abstract}

A mudança do ecossistema natural para o agroecossistema provoca alterações profundas nas propriedades químicas, físicas e biológicas dos solos (SÁ 1993), reduzindo drasticamente a quantidade de resíduos vegetais e alterando completamente as características do solo (BRADY 1984).

O plantio direto é um sistema de semeadura no qual a semente é colocada diretamente no solo não revolvido, através de um pequeno sulco ou cova de profundidade e largura suficientes para garantir uma boa cobertura e contato da semente com o solo (DERPSCH 1984). Portanto retém os resíduos vegetais na superfície, imitando assim os ecossistemas naturais: a estrutura do solo permanece, a temperatura e umidade são mais moderadas e assim o habitat se torna mais favorável à fauna do solo (PERDUE \& CROSSLEY 1989).

1) Curso de Pós-Graduação em Engenharia Florestal, Setor de Ciências Agrárias, Universidade Federal do Paraná. Caixa Postal 2959, 80001-970 Curitiba, Paraná, Brasil.

2) DCA/CEUD, Universidade Federal de Mato Grosso do Sul. Caixa Postal 533, 79804-970 Dourados, Mato Grosso do Sul, Brasil.

3) Departamento de Estatística, Universidade Federal do Paraná. Caixa Postal 19081, 81531-990 Curitiba, Paraná, Brasil. 
Segundo DUNGER (1983) os Collembola pertencem à mesofauna edáfica $(0,2$ a $2 \mathrm{~mm}$ de comprimento). Eles contribuem para a formação do solo de duas maneiras: primeiro, alimentando-se de material orgânico grosseiro que vai ser desdobrado em seus intestinos; segundo, produzindo fezes que vão ser adicionadas ao solo, podendo serem aproveitadas pelos demais organismos do solo (HALE 1971).

O presente trabalho tem como objetivo comparar a comunidade de Entomobryidae e Isotomidae (Collembola), entre plantio direto em três níveis de fertilidade, plantio convencional e um ecossistema natural (campo nativo).

\section{MATERIAL E MÉTODOS}

O presente trabalho foi conduzido em diversas propriedades de agricultores, cooperados da Cooperativa Agropecuária Batavo Ltda., região sul do Paraná, Segundo Planalto Paranaense, no município de Ponta Grossa ( $25^{\circ} 13^{\prime} \mathrm{S}$ e $\left.50^{\circ} 01^{\prime} \mathrm{W}\right)$.

Os solos das áreas pesquisadas foram todos caracterizados como Latossolo Vermelho-Escuro (EMBRAPA-SNLCS 1981), cujas características químicas e físicas são apresentadas na tabela I.

Tabela I. Análise química e física dos solos das áreas experimentais: plantio direto em área de baixa (A), média (B) e alta fertilidade (C), plantio convencional (D) e ecossistema natural (campo nativo) (E). Ponta Grossa, Paraná, setembro de 1993.

\begin{tabular}{|c|c|c|c|c|c|c|c|c|c|c|c|}
\hline \multirow{2}{*}{ Tratamentos } & $\mathrm{pH}$ & $\mathrm{Al}^{+3}$ & $\mathrm{H}+\mathrm{Al}$ & $\mathrm{Ca}^{+2}$ & $\mathrm{Mg}^{+2}$ & $\mathrm{~K}^{+}$ & \multirow[t]{2}{*}{$\begin{array}{c}P \\
(\mathrm{ppm})\end{array}$} & \multirow[t]{2}{*}{$\begin{array}{c}C \\
(\%)\end{array}$} & \multirow[t]{2}{*}{$\begin{array}{c}\text { Areia } \\
(\%)\end{array}$} & \multirow[t]{2}{*}{$\begin{array}{l}\text { Silte } \\
(\%)\end{array}$} & \multirow[t]{2}{*}{$\begin{array}{c}\text { Argila } \\
(\%)\end{array}$} \\
\hline & $\mathrm{CaCl}_{2}$ & \multicolumn{5}{|c|}{$\mathrm{meq} / 100 \mathrm{~cm}^{3}$ de solo } & & & & & \\
\hline A & 5,6 & 0,0 & 3,6 & 4,4 & 2,7 & 0,46 & 10 & 4,9 & 52 & 14 & 34 \\
\hline B & 6,2 & 0,0 & 2,9 & 7,5 & 3,3 & 0,35 & 27 & 4,4 & 48 & 20 & 32 \\
\hline C & 6,3 & 0,0 & 3,1 & 7,5 & 3,9 & 0,65 & 11 & 4,7 & 28 & 32 & 40 \\
\hline$D$ & 5,4 & 0,0 & 4,6 & 4,2 & 2,5 & 1.05 & 11 & 3,8 & 44 & 14 & 42 \\
\hline$E$ & 4,4 & 0,8 & 7,7 & 1,6 & 1,9 & 0,29 & 2 & 3,8 & 26 & 22 & 52 \\
\hline
\end{tabular}

O clima desta região, conforme FIAPAR (1978) é, segundo a classificação de Koeppen, classificado como Cfb, isto é, subtropical úmido, mesotérmico, verões frescos, geadas severas demasiadamente freqüentes e sem estação seca. A precipitação anual está em torno de 1400 a $1600 \mathrm{~mm}$, umidade relativa de $80 \%$ a $85 \%$ e a temperatura média anual de 17 a $18^{\circ} \mathrm{C}$. A precipitação e a temperatura média mensal durante o período do experimento encontram-se na figura 1.

No campo foi utilizado um delineamento de blocos ao acaso, com cinco tratamentos e três repetições. Em cada tratamento foi marcada, ao acaso, uma área de $45 \times 45 \mathrm{~m}$. Dentro destas áreas, foram marcados, também ao acaso, três blocos (repetições) de $3 \mathrm{~m} \times 3 \mathrm{~m}$. Estes tratamentos constituíram-se de plantio direto em três níveis de fertilidade, plantio convencional e um ecossistema natural (campo nativo). O cronograma de manejo de cada uma das áreas está descrito na tabela II.

Os Collembola foram estudados com base em amostragens feitas no período de 21 de outubro de 1992 a 29 de setembro de 1993. Foram realizadas no total 13 amostragens, com período aproximado entre elas de quatro semanas. A cada data efetuou-se três amostragens por parcela, perfazendo um total de nove amostras por tratamento e 45 amostras a cada data. 


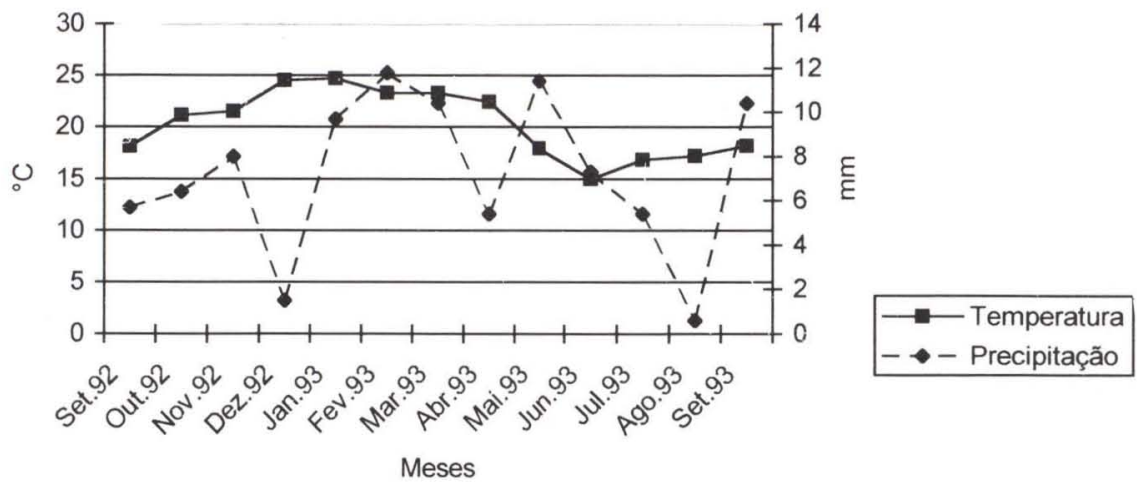

Fig. 1. Temperatura média diária $\left({ }^{\circ} \mathrm{C}\right)$, e precipitação média diária $(\mathrm{mm})$, registrada no município de Ponta Grossa, Paraná, de setembro de 1992 a setembro de 1993.

A coleta de amostras e a extração dos Collembola foi feita com o auxílio do método do Funil de Berlese modificado (diâmetro de $4 \mathrm{~cm}$ e profundidade de $5 \mathrm{~cm}$ ), descrito por SAUTTER \& SANTOS (1991). A identificação dos Collembola, ao nível de família, foi feita com o auxílio da chave de identificação proposta por PALÁciosVARGAS (1990), para a região Neotropical.

Os dados obtidos da mesofauna, devido à sua heterogeneidade, foram transformados em $\sqrt{x+0,5}$ (GERARD \& BERTHET 1966), sendo depois, submetidos à análise de variância (F-teste), e as médias comparadas pelo teste de Duncan ao nível de $10 \%$ de probabilidade, conforme COCHRAN \& CoX (1957)

\section{RESULTADOS E DISCUSSÃO}

\section{Flutuação populacional da comunidade de Entomobryidae}

A figura 2 mostra a flutuação populacional dos Entomobryidae. Pode-se observar que os tratamentos de plantio direto em área de baixa e média fertilidade, tiveram dois picos populacionais no ano: o primeiro no verão, e o segundo no inverno, o que concorda com BZUNECK \& SANTOS (1991). Isto ocorreu pelo fato de, nestas épocas, as culturas estarem na fase final de seu desenvolvimento (Tab. II), o que significou um ambiente propício para os Entomobryidae, em termos de temperatura e umidade, e sem distúrbios de ordem mecânica no ambiente (LORING et al. 1981). A população de Entomobryidae no plantio direto em área de alta fertilidade mostrou um pico populacional destacado, durante o período do experimento, abrangendo o verão e parte do outono. O ecossistema natural teve uma flutuação populacional inicial maior que os demais tratamentos, porém a partir de maio constatou-se uma grande queda devido à queima da vegetação (Tab. II), com rápido restabelecimento da população, a medida que houve o restabelecimento da vegetação (OLIVEIRA \& FRANKLIN 1993). Já o plantio convencional teve uma flutuação populacional constante, porém, com baixas densidades. Somente um pico foi verificado: no inverno. 


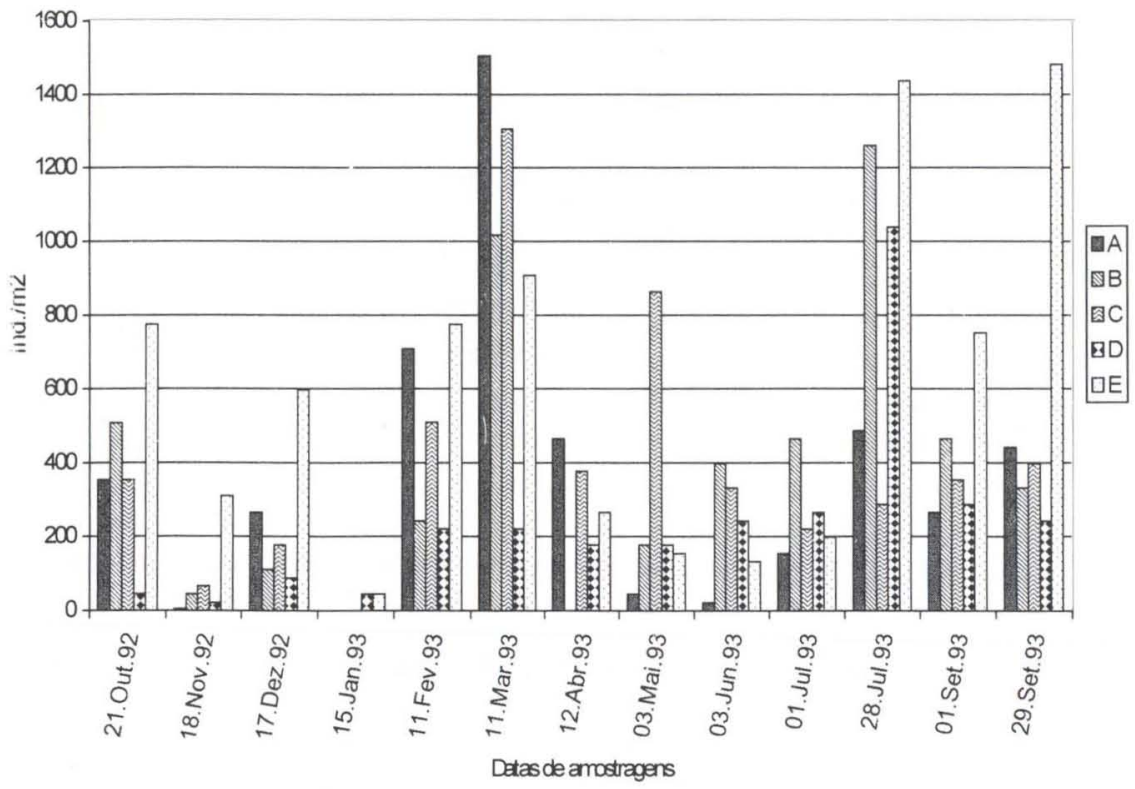

Fig. 2. Flutuação populacional da comunidade de Entomobryidae em plantio direto em área de baixa (A), média (B) e alta fertilidade (C), plantio convencional (D) e um ecossistema natural (campo nativo) (E). Ponta Grossa, Paraná, 1992/1993.

Tabela II. Cronograma de manejo conduzido no plantio direto em área de baixa fertilidade (A), média (B) e alta fertilidade (C), plantio convencional (D), e um ecossistema natural (campo nativo) (E), durante o período do experimento. Ponta Grossa, Paraná, 1992/1993.

\begin{tabular}{|c|c|c|}
\hline Tratamentos & Data & Operaçăo \\
\hline \multirow[t]{6}{*}{$A$} & $20-V-92$ & Plantio do trigo (variedade IAPAR-42) \\
\hline & $26-X-92$ & Colheita do trigo \\
\hline & $27-X-92$ & Plantio do soja (variedade $F-10$ ) \\
\hline & $14-I V-93$ & Colheita do soja \\
\hline & $16-I V-93$ & Plantio da aveia \\
\hline & VII-93 & Corte da aveia para silagem pré-secada \\
\hline \multirow[t]{6}{*}{ B } & VIII-92 & Incorporaçăo da ervilhaca ao solo \\
\hline & $29-1 X-92$ & Plantio do milho (veriedade P-3069) \\
\hline & Inicio III-93 & Colheita do milho \\
\hline & $10-111-93$ & A palha do milho é triturada \\
\hline & $24-111-93$ & Plantio do triticale \\
\hline & Inicio VII-93 & Corte do triticale para silagem pré-secada \\
\hline \multirow[t]{8}{*}{ C } & $17-\mathrm{VI}-92$ & Plantio do trigo (variedade BR-23) \\
\hline & $10-X \mid-92$ & Colheita do trigo \\
\hline & $17-\mathrm{XI}-92$ & Plantio do soja (variedade BR37) \\
\hline & $14-I V-93$ & Colheia do soja \\
\hline & $17-I V-93$ & Plantio da aveia \\
\hline & $28-1 V-93$ & Paraquat (200 g.i.a./ha) para dessecação total \\
\hline & $29-1 V-93$ & Plantio de Melilotus sp. a lanço/incorporaçăo com grade \\
\hline & $17-1 X-93$ & Plantio do milho \\
\hline \multirow[t]{6}{*}{ D } & VIII-92 & Incorporação da ervilhaca ao solo \\
\hline & $29-1 X-92$ & Plantio do milho (variedade P-3069) \\
\hline & Inicio III-93 & Colheita do milho \\
\hline & $10-111-93$ & A palha do milho é triturada \\
\hline & $24-111-93$ & Plantio do triticale \\
\hline & Inicio VII-93 & Corte do triticale para silagem pré-secada \\
\hline$E$ & Final IV-93 & Queima acidental da vegetação \\
\hline
\end{tabular}


A tabela III mostra a densidade média dos Entomobryidae, amostrados nos cinco tratamentos, resultados pelo teste de Duncan ao nível de $10 \%$ de probabilidade. Em sete das 13 épocas de amostragem foram constatadas diferenças estatísticamente significativas. Nas amostragens de outubro, novembro, fevereiro, final de julho e final de setembro, há uma predominância, em número de indivíduos, do ecossistema natural sobre os demais tratamentos. Isto pode ser explicado pela maior estabilidade ambiental neste sistema. Estes resultados contrariam os dados encontrados por WINTER et al. (1990), os quais relatam serem os Entomobryidae mais numerosos no plantio direto, do que num campo. Em abril pode-se notar uma co-dominância do ecossistema natural e do plantio direto. E em maio, o plantio direto em área de alta fertilidade se sobressai e há uma queda no ecossistema natural. Isto se dá pela queima generalizada ocorrida neste (Tab. II) (LUXTON 1982; OLIVEIRA \& FRANKLIN 1993).

Tabela III. Entomobryidae amostrados nos cinco tratamentos: plantio direto em área de baixa (A), média (B) e alta fertilidade (C), plantio convencional (D) e um ecossistema natural (campo nativo) (E). Média de nove amostras, expressa em número de individuos por $\mathrm{m}^{2}$. Ponta Grossa, Paraná, 1992/1993.

\begin{tabular}{|c|c|c|c|c|c|c|}
\hline Datas de amostragens & A & B & C & $\mathrm{D}$ & $E$ & Média \\
\hline $21-x-92$ & $354 b c$ & $508 a b$ & $354 b c$ & $44 c$ & $774 a$ & 407 \\
\hline $18-x \mid-92$ & $44 b$ & $44 b$ & $66 \mathrm{~b}$ & $22 b$ & $310 a$ & 97 \\
\hline $17-X \| 1-92$ & $265 a$ & $111 a$ & $177 a$ & $88 a$ & $597 a$ & 248 \\
\hline $15-1-93$ & Oa & Oa & Oa & $44 a$ & $44 a$ & 17 \\
\hline $11-11-93$ & $708 a$ & $243 b$ & $509 a b$ & $221 b$ & $774 a$ & 491 \\
\hline $11-|| \mid-93$ & $1504 a$ & $1017 a$ & $1305 a$ & $221 a$ & $907 a$ & 991 \\
\hline $12-I V-93$ & $464 a$ & $\mathrm{Ob}$ & $376 a$ & $177 b$ & $265 a b$ & 256 \\
\hline $3-V-93$ & $44 b$ & $177 b$ & $862 a$ & $177 b$ & $155 b$ & 283 \\
\hline $3-V 1-93$ & $22 a$ & $398 a$ & $332 a$ & $243 a$ & $133 a$ & 226 \\
\hline $1-V I I-93$ & $155 a$ & $464 a$ & $221 \mathrm{a}$ & $265 a$ & $199 a$ & 261 \\
\hline $28-V \| 1-93$ & $486 b$ & $1260 \mathrm{ab}$ & $287 c$ & $1039 a b$ & $1437 a$ & 901 \\
\hline $1-\mid X-93$ & $265 a$ & $464 a$ & $354 a$ & $287 a$ & $752 a$ & 424 \\
\hline $29-1 X-93$ & $442 b$ & $332 b$ & $398 b$ & $243 b$ & $1481 a$ & 579 \\
\hline Média & 366 & 386 & 403 & 236 & 602 & 399 \\
\hline
\end{tabular}

*) Médias, da mesma linha, seguidas pela mesma letra, não diferem significativamente pelo teste de Duncan, ao nível de $10 \%$ de probabilidade.

Os números finais revelam uma maior densidade no ecossistema natural, seguido pelos tratamentos de plantio direto e, por fim, o plantio convencional, o que concorda com os dados obtidos por WiNTER et al. (1990).

A implantação das culturas nos dois sistemas de preparo de solo (Tab. II), ocasionaram quedas na comunidade, também verificadas por LORING et al. (1981), MALLOW et al. (1985) e BZUNECK \& SANTOS (1991).

\section{Flutuação populacional da comunidade de Isotomidae}

A figura 3 mostra a flutuação populacional de Isotomidae. No plantio direto em área de alta fertilidade e no ecossistema natural, não houve uma grande flutuação populacional, porém esta manteve-se com baixas densidades. O plantio direto em área de baixa fertilidade teve um pico populacional destacado no verão, e outro menor no inverno, quando criou-se um microclima favorável (LORING et al. 1981); assim como o plantio convencional teve um pico populacional na safra de inverno. Já no plantio direto em área de média fertilidade, a população de Isotomidae teve dois picos populacionais: um no verão e outro no inverno. 


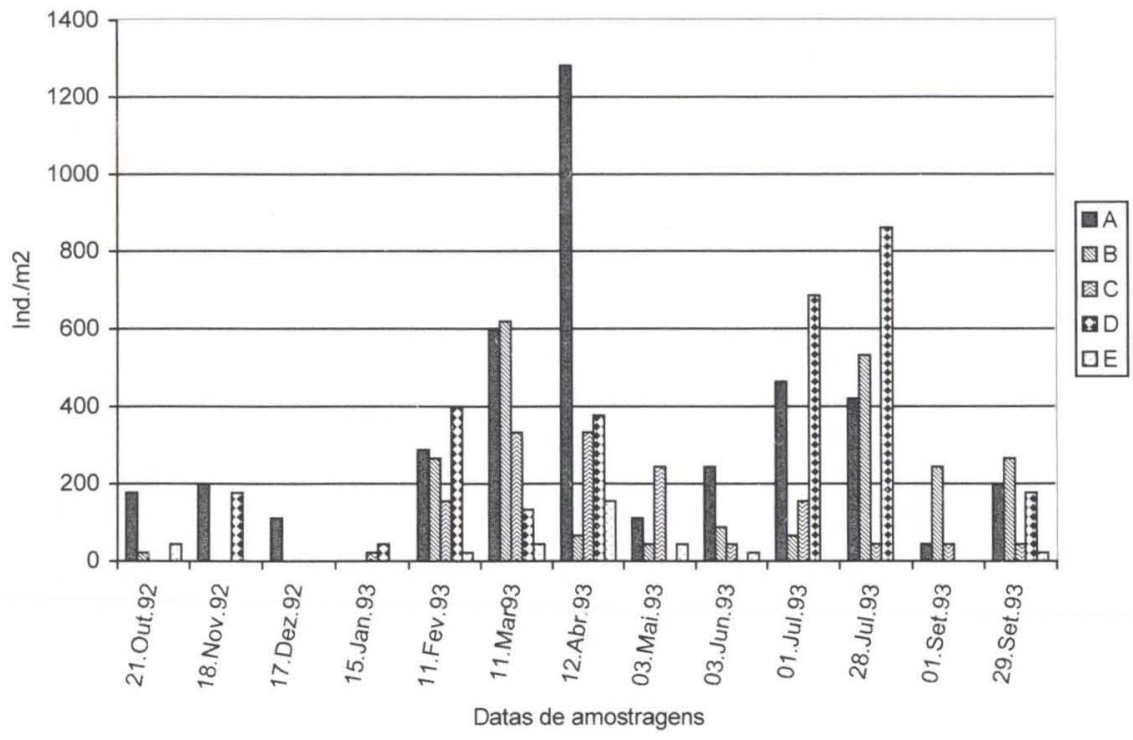

Fig. 3. Flutuação populacional da comunidade de Isotomidae em plantio direto em área de baixa (A), média (B) e alta fertilidade (C), plantio convencional (D) e um ecossistema natural (campo nativo) (E). Ponta Grossa, Paraná, 1992/1993.

A tabela IV mostra a densidade média dos Isotomidae, amostrados nos cinco tratamentos e os resultados comparados pelo teste de Duncan ao nível de $10 \%$ de probabilidade. Em nove das 13 amostragens realizadas, encontrou-se diferenças estatísticamente significativas entre os tratamentos. Em fevereiro e no início e final de julho, os resultados do plantio convencional foram superiores aos demais. Já em outubro, dezembro, março, abril, maio e final de setembro, os resultados dos dados do plantio direto foram superiores aos do plantio convencional e ao ecossistema natural, dados estes que estão de acordo aos encontrados por BLUMBERG \& Crossley (1983). Isto se deve, provavelmente, ao fato de nestas épocas ter havido baixas precipitações (Fig. 1), obrigando aos Isotomidae migrarem para camadas mais profundas do solo nestes tratamentos, portanto não sendo amostrados no plantio convencional e no ecossistema natural, já que o plantio direto mantém mais estáveis as condições de umidade e temperatura do solo (DERPSCH et al. 1991).

A densidade populacional média final dos Isotomidae em cada tratamento, revela que o plantio direto em área de baixa fertilidade foi superior, vindo em seguida o plantio convencional, plantio direto em área de média fertilidade, plantio direto em área de alta fertilidade, e, por fim, o ecossistema natural. O que concorda com WINTER et al. (1990).

\section{CONCLUSÕES}

Os Entomobryidae e Isotomidae foram encontrados em maior número, quando o estágio de desenvolvimento das culturas era adiantado, formando uma boa cobertura do solo. 
Tabela IV. Isotomidae (Collembola) amostrados nos cinco tratamentos: plantio direto em área de baixa (A), média (B) e alta fertilidade (C), plantio convencional (D) e um ecossistema natural (campo nativo) (E). Média de nove amostras, expressa em número de indivíduos por $\mathrm{m}^{2}$. Ponta Grossa, Paraná, 1992/1993.

\begin{tabular}{|c|c|c|c|c|c|c|}
\hline Datas de amostragens & A & B & C & $D$ & $E$ & Média \\
\hline $21-X-92$ & $177 a$ & $\mathrm{Ob}$ & $\mathrm{Ob}$ & $\mathrm{Ob}$ & $44 b$ & 49 \\
\hline $18-X \mid-92$ & $199 a$ & Oa & Oa & $177 a$ & $\mathrm{Oa}$ & 75 \\
\hline $17-X I I-92$ & $111 a$ & $a b$ & $\mathrm{Ob}$ & $O b$ & $\mathrm{Ob}$ & 22 \\
\hline $15-1-93$ & Oa & $0 a$ & $22 a$ & $44 a$ & $\mathrm{Oa}$ & 13 \\
\hline $11-11-93$ & $287 a b$ & $265 a b$ & $155 a b$ & $398 a$ & $22 b$ & 225 \\
\hline $11-111-93$ & $597 a$ & $619 a$ & $332 a b$ & $133 a b$ & $44 b$ & 345 \\
\hline $12-I V-93$ & $1282 a$ & $66 b$ & $332 b$ & $376 a b$ & $155 b$ & 442 \\
\hline $3-V-93$ & $111 b$ & $44 b c$ & $243 a$ & Oc & $44 \mathrm{bc}$ & 88 \\
\hline $3-\mathrm{VI}-93$ & $243 a$ & $88 a$ & $44 a$ & $0 \mathrm{a}$ & $22 a$ & 79 \\
\hline $1-V I I-93$ & $464 a b$ & $66 a b$ & $155 a b$ & $685 a$ & $\mathrm{Ob}$ & 274 \\
\hline 28-VII-93 & $420 \mathrm{ab}$ & $531 a b$ & $44 a b$ & $862 a$ & $\mathrm{Ob}$ & 371 \\
\hline $1-\mid X-93$ & $44 a$ & $243 a$ & $44 a$ & $\mathrm{Oa}$ & $\mathrm{Oa}$ & 66 \\
\hline $29-1 X-93$ & $199 a b$ & $265 a$ & $44 b c$ & $177 a b c$ & $22 c$ & 141 \\
\hline Média & 318 & 170 & 109 & 219 & 27 & 169 \\
\hline
\end{tabular}

*) Médias, da mesma linha, seguidas pela mesma letra, não diferem significativamente pelo teste de Duncan, ao nivel de $10 \%$ de probabilidade.

Os Entomobryidae e Isotomidae foram muito sensíveis à falta de chuva em todos os tratamentos, sendo que a maior queda foi no plantio convencional.

As comunidades de Entomobryidae e Isotomidae diminuiram muito com a queima da vegetação no ecossistema natural, com restabelecimento relativamente rápido, e este ligado ao restabelecimento da vegetação.

A implantação das culturas, tanto no plantio direto, quanto no convencional, foi uma das principais causas da queda da população.

\section{REFERÊNCIAS BIBLIOGRÁFICAS}

BLUmBerG, A.Y.; D.A. Crossley JR. 1983. Comparison of soil surface arthropod populations in conventional tillage, no-tillage and old field systems. AgroEcosystems 8: 247-253.

BRADY, B. 1984. The nature and properties of soils. New York, Macmillan Publishing Company, $9^{\text {th }}$ ed., 750 p.

BZUNECK, H.L.; H.R. DOS SANTOS. 1991. Efeitos de dois sistemas de preparo de solo e de sucessões de culturas na população de colêmbolos Dicranocentrus spp.

Rev. Set. Ciênc. Agrár. 11 (1-2): 231-235.

Cochran, W.G.; G.M. CoX. 1957. Experimental designs. New York, John Wiley $\&$ Sons Inc., $2^{\text {nd }}$ ed., 595 p.

DERPSCH, R. 1984. Programa de manejo e conservação do solo, p.13-46. In: P.V. TORRADO \& R.R. AlOISI (Ed.). Plantio direto no Brasil. Campinas, Fundação Cargill, 124p.

DERPSCH, R.; C.H. Roth; N. SidiRAS; U. KoEPKE. 1991. Controle da erosão no

Paraná, Brasil: Sistemas de Cobertura de solo, plantio direto e preparo conservacionista do solo. Eschborn, GTZ, Iapar, 272p.

Dunger, W. 1983. Tiere im Boden. Die Neue-Brehm Buecherei 327. Wittemberg, A. Ziemsen Verlag, 287p. 
EMBRAPA-SNLCS. 1981. Levantamento de Reconhecimento dos Solos do Estado do Paraná. Curitiba, Embrapa/SNLCS, 1 mapa, escala 1:6.000.000.

FIAPAR. 1978. Cartas Climáticas do Estado do Paraná. Londrina, Fiapar, 38p.

Gerard, G.; P. BerTHET. 1966. A statistical study of microdistribution of Oribatei (Acari). Part II: The transformation of the data. Oikos 17: 142-149.

Hale, W.G. 1971. Colembolos, p.463-477. In: A. Burges \& F. Raw (Eds). Biologia del suelo. Barcelona, Omega.

LORING, S.J.; R.J. SNIDER \& L.S. ROBERTSON. 1981. The effects of three tillage practices on Collembola and Acarina populations. Pedobiologia 22: 172-184.

LUXTON, M. 1982. Studies on the invertebrate fauna of New Zealand peat soils. I. General survey of the sites. Rev. Ecol. Biol. Sol. 19: 535-552.

MALLOW, D.; R.J. SNIDER \& L.S. ROBERTSON. 1985. Effects of different management practices on Collembola and Acarina in corn production systems. II. The effects of moaldboard plowing and Atrazine. Pedobiologia 28: 115-131.

OLIVEIRA, E.P. DE \& E. FrANKLIN. 1993. Efeito do fogo sobre a mesofauna do solo: recolonização em áreas queimadas. Pesquisa Agropecuária Brasileira 28 (3): 357-369.

PALÁCIOS-VARGAS, J.G. 1990. Diagnosis y clave para determinar las familias de los Collembola de la Región Neotropical. Manuales y Guias para el Estudo de Microartrópodos. México, Fac. Ciencias (UNAM), 15p.

Perdue, J.C. \& D.A. Crossley JR. 1989. Seasonal abundance of soil mites (Acari) in experimental agroecosystems: Effects of drought in no-tillage and conventional tillage. Soil \& Tillage Res. 15: 117-124.

SÁ, J.C. DE M. 1993. Manejo da fertilidade do solo no plantio direto. Castro, Fundação ABC, 96p.

SAUTTER, K.D. \& H.R. DOS SANTOS. 1991. Recuperação de solos degradados pela mineração de xisto, tendo como bioindicadores insetos da ordem Collembola. Ver. Set. Ciênc. Agrár. 11 (1-2): 85-91.

WINTER, J.P.; R.P. VORONEY \& D.A. AINSwORTH. 1990. Soil microarthropods in long term no-tillage and conventional tillage corn production. Can. Jour. Soil. Sci. 70: 641-653.

Recebido em 29.VIII.1997; aceito em 25.I.1999. 